\title{
Propuestas para un diccionario conceptual bilingüe para Ele y DaF: ¿Qué aportan los diccionarios de aprendizaje para situaciones de expresión escrita en lengua extranjera?
}

Meike Meliss

Universidad de Santiago de Compostela / Red de Lexicografía RELEX

Some proposals for a conceptual bilingual dictionary of German and Spanish as foreign languages.

\section{Abstract}

This paper presents some theoretical and methodological foundations of the research project DICONALE, which concerns the development of an online dictionary of verbal lexemes with a special conceptual-onomasiological access and a paradigmatic structure in response to studies which have shown that conventional dictionaries (both monolingual and bilingual), do not satisfy the specific needs of users involved in the production of texts in foreign language.

Keywords

bilingual lexicography, learner dictionaries, paradigmatic sense relations, syntagmatic relations.

\section{Introducción}

Esta aportación se centra en la relevancia de un tratamiento específico de la variedad y la variación léxica en determinadas obras lexicográficas a nivel inter- e intralingual para favorecer los contextos de la expresión (escrita) en alemán y/o español como lenguas extranjeras (en adelante: DaF y Ele). Partiendo de un análisis crítico de algunas obras lexicográficas seleccionadas para conocer su utilidad en el proceso de output en la lengua extranjera (en adelante: L2), se somete a discusión una propuesta lexicográfica innovadora elaborada en el marco del proyecto de investigación 
DICONALE $^{1}$ y que surge de las deficiencias lexicográficas en contextos de la producción lingüística en DaF y Ele.

A la hora de producir un texto en lengua extranjera - sea como expresión libre o vinculado al proceso de traducción - es de suma importancia, disponer del léxico adecuado junto con toda la información lexicológica relevante para verbalizar la intención comunicativa concreta en cuestión. Este estudio parte de la observación que tanto los procesos de búsqueda de lexemas adecuados - a veces incluso desconocidos en contextos de L2 - que lexicalicen o bien un concepto determinado en consonancia con la intención comunicativa del hablante L2 o que sirvan de equivalente de traducción, al igual que los mecanismos de selección del lexema adecuado entre la posible variedad léxica en la lengua meta, siguen caracterizándose por un tratamiento deficitario en la lexicografía actual de las lenguas que aquí nos interesan ( $c f r$. Meliss 2013a, 2013b, 2014a, 2014b). La oferta informativa para el adecuado y correcto uso del lexema seleccionado en la lengua meta L2 resulta igualmente insuficiente en el contexto de la producción en L2 y requiere un tratamiento más adaptado a la situación de uso que aquí está en el centro del interés. Por consiguiente, el capítulo 2 de esta aportación examinará la utilidad de diferentes diccionarios bi- y monolingües para los procesos de producción en L2. Como propuesta lexicográfica alternativa a los déficits que resultan del análisis, se presentarán en el capítulo 3 algunos aspectos relevantes del proyecto DICONALE. En consonancia con los objetivos aquí dibujados, se encuentran en el centro del interés de esta aportación (i) el proceso de búsqueda de lexemas adecuados para el proceso de producción en L2, (ii) los mecanismos de selección entre la posible variedad de expresión a nivel inter- e intralingual y (iii) la oferta informativa para su uso adecuado en procesos de producción en la respectiva L2. Los análisis se basan de modo ejemplar en algunas posibles verbalizaciones que existen en ambas lenguas para expresar el concepto PERCEPCIÓN AUDITIVA. A su vez, los fundamentos empíricos resultan de los estudios pertinentes basados en los corpus lingüísticos de referencia DeReKo/Cosmas II para el alemán y CREA para el español. ${ }^{2}$

2. El acercamiento a diferentes tipos de diccionarios en el proceso de la expresión (escrita) en contextos de L2: buscar - seleccionar - usar

A la hora de redactar un texto en una lengua extranjera y querer expresar determinados conceptos e ideas estamos sujetos a la búsqueda de verbalizaciones adecuadas, a la selección entre la variedad de posibilidades y finalmente a la necesidad de conocer las reglas para el uso correcto del lexema elegido.

1 Esta aportación se ha elaborado en el marco de los proyecto de investigación DICONALEestudios (Xunta de Galicia: IN.CI.TE: 10PXIB204188PR) y DICONALE-online (MINECOFEDER: FFI2012-32658) y en relación con la red lexicográfica RELEX (Xunta de Galicia/FEDER: CN2012/290).

2 Para la base empírica de DICONALE véase González Ribao (2014) (en este mismo tomo). 
Metodológicamente podemos enfrentarnos de diferente manera a la tarea. En contextos de L2 preferentemente se realiza la consulta mediante diccionarios bilingües y diccionarios monolingües pedagógicos. Es menos frecuente la consulta en obras lexicográficas específicas, como pueden ser los diccionarios de relaciones paradigmáticas y sintagmáticas o en obras con una perspectiva onomasiológica, ${ }^{3}$ ya que no suele estar disponible tal variedad de obras lexicográficas, al no ser que tengan un acceso en línea que permita al usuario una búsqueda gratuita, cómoda y rápida. Por este motivo se analizarán a continuación con especial atención las obras de consulta online que están a disposición del usuario que aquí se enfoca.

\subsection{Los diccionarios bilingües}

En los diccionarios bilingües de las lenguas en cuestión, la búsqueda y selección del lexema adecuado resulta a menudo dificultosa tanto por la escasa desambiguación semántica y sintáctica en caso de polisemia en la L2 como lengua de partida, como por la falta de criterios coherentes que restringen la selección en caso de equivalencias múltiples en la L2 como lengua de llegada. La falta de información lexicológica detallada y distintiva para la lengua de llegada dificulta además su correcto uso en caso de tratarse de L2 (Meliss 2011, 2013a, 2013b, 2014a). En el proceso de búsqueda de un lexema adecuado mediante la consulta en diccionarios bilingües se presentan a mayores las siguientes dos dificultades. A la hora de partir de la lengua materna (L1) solo podemos buscar lo que está lexicalizado en la L1 como lengua de partida y se nos "escapa" todo aquello para lo cual no existen verbalizaciones en la L1 pero si en L2. Los vacíos léxicos que quedan sobre todo plasmados en estudios contrastivos de paradigmas léxico-semánticos son un buen ejemplo de ello (González Ribao / Proost 2014; Proost 2007). Los diccionarios delimitan además el número de sus entradas según criterios comerciales y en consonancia con el tamaño y su usuario mediante determinados criterios, entre los cuales se puede mencionar la frecuencia de uso. De ese modo resulta evidente que determinados lexemas no aparecen en las obras de consulta y el número de equivalencias para un lexema en L2 como lengua de llegada sea igualmente restringido.

3 Los diccionarios onomasiológicos clásicos como son p. ej. para el alemán las obras de Dornseiff et al. $\left(2004^{8}\right)$ y de Wehrle / Eggers $\left(1961 / 1993^{17}\right)$ o el diccionario ideológico de Casares (1942/2011) para el español nos ofrecen una primera visión acerca de la variedad de posibles lexicalizaciones, pero no aportan datos que ayuden a la selección de uno u otro y no aseguran su correcto y adecuado uso en una situación de producción en L2, ya que se trata únicamente de listas de palabras ( $c f r$. Hausmann 1990a y b; Engelberg / Lemmnitzer 2001/20094; Meliss 2005). Además se trata de diccionarios específicos que en la situación aquí en cuestión no suelen estar disponibles. Un caso especial dentro de esta tipología son los diccionarios visuales, que gozan de más aceptabilidad en entornos de L2.

En este mismo volumen. 
Se puede afirmar, pues, que los diccionarios bilingües no siempre aportan la información adecuada para los procesos de búsqueda, selección y uso en el proceso de la producción lingüística en la L2 (cfr. Fuentes Morán 1997; Model 2010). Por ello, el usuario acude a menudo mediante los enlaces externos a la información que ofrecen los diccionarios en línea para así ampliar su consulta a diccionarios monolingües generales y/o diccionarios específicos, como son p. ej. los diccionarios de relaciones paradigmáticas. A modo de ejemplo es de mencionar que los diccionarios bilingües en línea de los portales de Leo y Pons ponen a disposición del usuario un amplio repertorio de recursos externos a obras lexicográficas y corpus lingüísticos para el español y el alemán (figuras 1-2). Pero en un contexto de L2 estas obras resultan a menudo inadecuadas, no solo por su complejidad informativa debido al hecho de seguir principalmente un enfoque dirigido hacia usuarios de L1 (Español: MM, DRAE, CLAVE, etc.; Alemán: OWID, DWDS, Wortschatz UniLeipzig, etc.) sino también debido a la falta de una preparación lexicográfica adecuada del usuario aquí en cuestión. ${ }^{5}$

\begin{tabular}{|c|c|}
\hline Links externos: diccionarios del español & Links externos: diccionarios del alemán \\
\hline $\begin{array}{l}\text { Links ZU Weiteren Informationen } \\
\text { Lexika/Enxyklopädien } \\
\text { Wikipedia } \\
\text { Definitionen/Erklärungen } \\
\text { Maria Moliner } \\
\text { Dicclonario panhispánico de dudas } \\
\text { DRAE - Diccionario de la Real Academia Espathola } \\
\text { Clave } \\
\text { Korpora/Belegsammlungen } \\
\text { Corpus de Referencia del Español Actual (CREA) } \\
\text { Bildwörterbücher } \\
\text { Diccionario visual } \\
\text { Synonyme/Antonyme } \\
\text { Santillana } \\
\text { Redewendungen/Sprichwörter } \\
\text { Retranero Bilingue, Inst Cervantes } \\
\text { Konjugation/Deklination } \\
\text { Cactus2000 } \\
\text { El Conjugador }\end{array}$ & 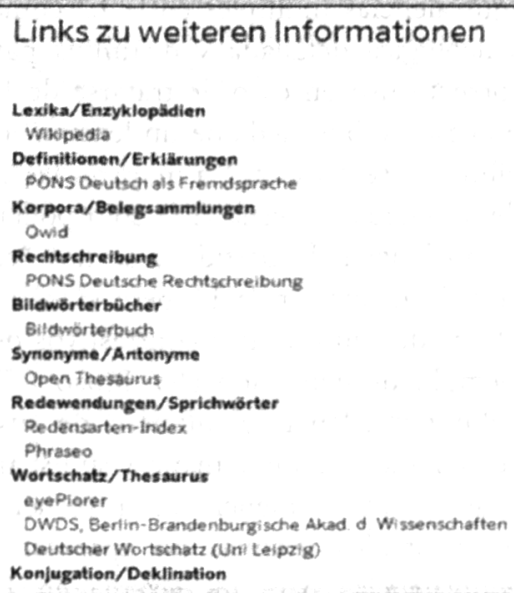 \\
\hline
\end{tabular}

Figura 1: Links externos del diccionario bilingüe alemán/español del portal de Pons para consultas en otras obras lexicográficas del español y del alemán

5 El usuario L2 que no ha recibido una instrucción previa no sabe, en general, aprovechar la cantidad de información modular que ofrecen portales como DWDS, Wortschatz UniLeipzig o cannoNet. 


\begin{tabular}{|l|l|l|l|}
\hline \multicolumn{4}{|l|}{ LEO: Informaciones adicionales } \\
\hline Fuente & Información sobre escuchar & Información sobre hören & Fuente \\
\hline LEO & $\begin{array}{l}\text { Pronunciación } \\
\text { escuchar }\end{array}$ & $\begin{array}{l}\text { Pronunciación } \\
\text { hören }\end{array}$ & LEO \\
\hline LEO & $\begin{array}{l}\text { Tabla de conjugación } \\
\text { escuchar }\end{array}$ & $\begin{array}{l}\text { Tabla de conjugación } \\
\text { hören }\end{array}$ & LEO \\
\hline $\begin{array}{l}\text { Real Academia } \\
\text { Española }\end{array}$ & $\begin{array}{l}\text { Definición } \\
\text { escuchar }\end{array}$ & $\begin{array}{l}\text { Definición } \\
\text { hören }\end{array}$ & DWDS \\
\hline & & $\begin{array}{l}\text { Conjugación } \\
\text { hören }\end{array}$ & canoonet \\
\hline
\end{tabular}

Figura 2: Links externos del diccionario bilingüe alemán/español en línea de Leo para consultas en otras obras lexicográficas del español y del alemán

2.2. La búsqueda, selección y uso en diccionarios específicos de relaciones paradigmáticas y sintagmáticos

2.2.1. Los diccionarios específicos de estructuración paradigmática permiten una búsqueda mediante la indicación de relaciones léxico-semánticas entre diferentes lexemas. A parte de las versiones clásicas en formato print, se puede acceder mediante diferentes portales lexicográficos a módulos que ofrecen información paradigmática de significado. ${ }^{6}$ Los diccionarios bilingües arriba mencionados (cap. 2.1.) ofrecen enlaces directos a alguno de estos recursos lexicográficos (figuras 1-2).? Pero tanto su formato clásico print como los correspondientes módulos de los portales lexicográficos mencionados, muestran poca información para la selección y el uso en contextos de producción en L2. ${ }^{8}$ Suelen ser sobre todo listas de palabras con diferentes recursos lingüísticos, escasea la información que facilite la selección de uno u otro lexema y carecen de información que pueda asegurar un correcto uso, ya que, en general, no son sinonimias distintivas. La posibilidad de ampliar la información mediante mecanismos de enlaces con otros módulos informativos del

6 Para el alemán son de especial mención los módulos de los siguientes portales y diccionarios online: elexiko, DWDS, Wortschatz Uni-Leipzig, cannoNet, woxikon, Wörterbuch für Synonyme, OpenThesaurus, etc.. Para el español se pueden nombrar entre otros el "diccionario de sinónimos" diccionarios.com y la información ofrecida en WordReference.

7 OWID ofrece enlaces externos a cannoNet, Wortschatz Uni-Leipzig ofrece enlaces externos a la versión electrónica del diccionario onomasiológico de Dornseiff et al. $\left(2004^{8}\right)$ y DWDS enlaza con OpenThesaurus. El portal WordReference enlaza la información con los materiales lexicográficos de la editorial española Espasa-Calpe así como con el DRAE y el portal diccionarios.com enlaza con materiales de las editoriales Vox y Larousse.

8 Véanse los siguientes ejemplos para la información sobre relaciones paradigmáticas de sentido en ambas lenguas: Alemán: (a) Módulo informativo de DWDS para la entrada zuhören, (b) secuencia del portal canooNet para la entrada zuhören. Español: (a) escuchar del portal diccionarios.com ("diccionario de sinónimos"), (b) oir del portal WordReference. 
mismo portal o incluso mediante enlaces externos facilita, sin embargo, la ampliación de la información y proporciona al usuario una considerable ventaja frente a los diccionarios clásicos print, pero requiere por parte del usuario un elevado conocimiento sobre los diferentes recursos lexicográficos online para no correr el peligro de perder la orientación (lost in hyperspace: Haß / Schmitz 2010: 4). ${ }^{9}$ Con alguna excepción no existen hasta el momento obras lexicográficas con acceso en línea que se basan en amplios estudios de paradigmas léxico-semánticos complejos $^{10}$ (cfr. Meliss 2005).

2.2.2. Los diferentes diccionarios específicos de relaciones sintagmáticas y especialmente los diccionarios de construcciones, colocaciones y de régimen verbal deben ofrecer al interesado sobre todo la información pertinente para el correcto uso. Si bien existen para ambas lenguas diferentes tipos de diccionarios sintagmáticos en formato print,${ }^{11}$ hay que afirmar que para la situación que aquí nos interesa, escasea la oferta en la red. Ninguno de los links externos ofrecidos a diccionarios arriba mencionados (cap. 2.1.: figuras 1-2) lleva al usuario a una información explícitamente sintagmática.

Únicamente un buen conocedor de los diferentes recursos electrónicos sabe, por ejemplo, que el diccionario de valencias verbales del alemán VALBU (2004) tiene una versión electrónica $E$-Valbu a la que se accede desde la página Web del IDS de Mannheim, que a través del portal OWID se accede al diccionario elexiko y a los módulos de colocaciones, construcciones y gramática con la información pertinente sobre la combinatoria, y que detrás de los links externos a los diccionario DWDS y Wortschatz Uni Leipzig se esconde p. ej. información sintagmática en torno al potencial combinatorio mediante información generada automáticamente y visualizado con los "Wortprofile". En caso de acceder a esta información resulta por otra parte de difícil interpretación en entornos $\mathrm{DaF}$ sin haber tenido una instrucción lexicográfica previa.

9 El acceso a la información de WordNet, GermaNet, WordNetespañol y EuroWordNet etc. está fuera del alcance de un usuario de $\mathrm{DaF}$ o Ele. La información ofrecida en estas redes requiere un proceso de adaptación para los entornos DaF y Ele.

10 Merece especial atención el diccionario en línea que trata los verbos de comunicación del alemán (KV-online), alojado en el portal lexicográfico OWID del IDS de Mannheim. Esta versión online se basa en la versión print de Harras et al. (2004, 2007). Para el español podemos mencionar el prototipo $\mathrm{DiCe}$ ( diccionario de colocaciones del español) que ofrece las colocaciones en torno a los "sustantivos de sentimiento" (Alonso Ramos 2004, 2008).

11 De especial mención son los diccionarios con información sintagmática que facilitan al usuario la información adecuada para sus necesidades de producción y por tanto la información sobre la correcta y adecuada combinatoria, como son p. ej., para el alemán los diccionarios de colocaciones (p. ej.: Quasthoff 2011; Duden Stilwörterbuch 2010 ${ }^{9}$ ) y los diccionarios de valencias (p. ej. VALBU 2004). Para el español destacan las obras lexicográficas de Seco et al. (DEA: 1999) y Cuervo (1953/1998) con información sobre el régimen verbal, los diccionarios combinatorios (Bosque 2004) y el proyecto lexicográfico para la elaboración del Diccionario Coruña (Porto Dapena et al. 2007, 2008). 
Para la información sintagmática del español estamos igualmente ante una situación compleja. No está disponible en línea ninguna de las obras lexicográficas con un enfoque específicamente sintagmático y proyectos como el diccionario de colocaciones del español (DiCE: Alonso Ramos 2004, 2008), el diccionario de aprendizaje del español como lengua extranjera (DAELE: Battaner Arias 2008; Bernal / Renau 2010) o el diccionario contrastivo de valencias verbales: españolalemán (DCVVEA: Domínguez Vázquez et al. 2008a, 2008b) hasta el momento son o bien únicamente prototipos o bien abarcan un número reducido de entradas.

2.2.3. Los diccionarios monolingües pedagógicos / de aprendizaje: Ante la situación arriba descrita, los diccionarios monolingües pedagógicos / de aprendizaje para $\mathrm{DaF}^{12}$ y $\mathrm{Ele}^{13}$ pretenden ofrecer una alternativa tanto a los diccionarios bilingües como a los diccionarios monolingües generales. Sin embargo, la búsqueda de lexemas desconocidos en diccionarios monolingües de aprendizaje de L2 lleva a pocos resultados satisfactorios para la situación que aquí nos preocupa, ya que dichos diccionarios siguen un orden alfabético y una perspectiva semasiológica que no favorece la tarea de buscar y encontrar un lexema de antemano desconocido. A excepción del diccionario pedagógico alemán de Kempcke et al. (1999), ${ }^{14}$ la lexicografía pedagógica monolingüe para entornos de DaF y Ele ha reaccionado hasta el momento de forma insuficiente a los problemas específicos de búsqueda y selección de un lexema adecuado a la hora de producir un texto en L2, ya que no ofrecen alternativas reales a las consultas semasiológicas por orden alfabético. ${ }^{15} \mathrm{De}$ allí que el interés de la consulta lexicográfica mediante este tipo de obras pedagógicas para los fines aquí en cuestión, se centra primordialmente en la información que pueden ofrecer éstas para el correcto uso.

Se ha observado en la última década un enorme esfuerzo - sobre todo en el ámbito de $\mathrm{DaF}$ - para incorporar información de uso que se materializa por un lado en la información acerca de las relaciones paradigmáticas de sentido que aportan

12 En este estudio se ha delimitado el análisis a los diccionarios DaF de Götz et al. (LGWBDaF: 2007, 2010) y de Kempcke et al. (1999). Para más información sobre diccionarios de aprendizaje para DaF remito a los estudios de Meliss (2013b, 2014a, 2014b).

13 Se ha delimitado aquí el análisis a los diccionarios Ele de Salamanca (DSA: 2006), de Alcalá (DA: 1995) con libre acceso restringido desde el portal <www.diccionarios.com> y el análisis de DAELE. Otros diccionarios para el uso en situaciones de Ele, como puede ser el diccionario Clave con libre acceso online o el DEE (2002) no se han tenido en cuenta.

14 Kempcke et al. (1999) combina en su diccionario para DaF el proceso de búsqueda desde una perspectiva semasiológica con una perspectiva onomasiológica. A falta de una preparación lexicográfica adecuada, el usuario no se suele aprovechar de esta oferta informativa. La representación de las interrelaciones entre el léxico está además muy limitada en el marco de un diccionario print (Roelcke 2002).

15 En este lugar hay que mencionar el Diccionario Coruna (Porto Dapena et al. 2007, 2008) que, aunque no se haya concebido expresamente como diccionario para Ele, presenta como novedad un carácter reversible. Se propone añadir a la parte semasiológica una parte onomasiológica. La interrelación de ambas partes se expone a modo de ejemplo con los verbos relacionados con la alimentación (Porto Dapena et al. 2007). 
recursos para la variedad estilística de expresión (Wolski 2002) y por otro en la información combinatoria. ${ }^{16} \mathrm{El}$ diccionario para $\mathrm{DaF}$ analizado aquí de forma ejemplar (figura 3) ofrece información a nivel semántico (relaciones paradigmáticas de significado y rasgos semánticos distintivos) que permite la selección de una u otra acepción junto con la información combinatoria que abarca el rasgo sintáctico de la transitividad, el esquema actancial, el contorno sintáctico-semántico y colocaciones frecuentes.

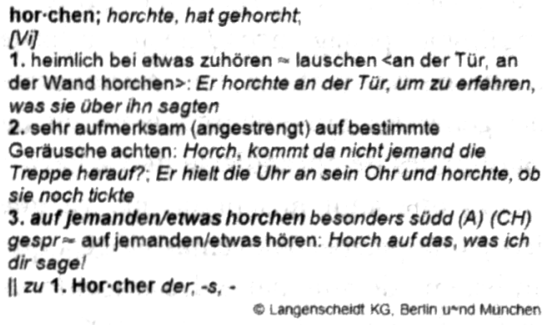

\begin{tabular}{|c|c|c|c|}
\hline & $\begin{array}{l}\text { Relaciones } \\
\text { paradigmáticas de } \\
\text { significado: }\end{array}$ & $\begin{array}{l}\text { Rasgos } \\
\text { semanticos } \\
\text { distintivos \& } \\
\text { registro }\end{array}$ & $\begin{array}{l}\text { Informacion sobre la } \\
\text { combinatoria \& } \\
\text { contorno sintactico- } \\
\text { semántico }\end{array}$ \\
\hline 1 & $\begin{array}{l}\text { o zuhoren } \\
\text { lauschen }\end{array}$ & heirnich & $\begin{array}{l}\text { Vi } \\
\text { Col: }<\text { an der } \\
\text { Tur } W \text { and }>> \\
\text { Esq }<? \geqslant>\end{array}$ \\
\hline 2 & $\approx$ achten auf & aufmerksam & $\begin{array}{l}V_{1} \\
\text { Esqs }<n n>\end{array}$ \\
\hline 3 & $\begin{array}{l}=\text { hören auf } \\
\text { jemanden/etwas }\end{array}$ & $\begin{array}{l}\text { R: } \\
\text { sodd } \\
\text { (A) }(\mathrm{CH})\end{array}$ & $\begin{array}{l}\text { Esq auf jemanden ! } \\
\text { efwas }\end{array}$ \\
\hline
\end{tabular}

Figura 3: horchen de LGWBe-DaF (2007) y resumen esquemático de la información

Los diccionarios de Ele, y concretamente los diccionarios de Salamanca (DSA 2006) y de Alcalá ( $D A$ 1995) ofrecen información a nivel semántico mediante perífrasis y relaciones paradigmáticas de significado que facilitan la selección de una u otra acepción. El uso de una u otra acepción se describe además mediante información sobre la combinatoria que abarca la transitividad, indicaciones sobre el esquema actancial y el contorno sintáctico- semántico. Las colocaciones más frecuentes, sin embargo, no se contemplan (figuras 4-5):

escuchar $v, t r .1$ Poner e una persona , atención para oir [una cosa]: Escuchamos Las noticias en la radio. 2 Dejarse influir s una persona > por las palabras o consejos de lotra persona]: Le be estado diciendo que no lo baga, pero no me ha escucbado, 3 amte. Oí, percibir sonidos. If $v$, intr. 4 Aplicar cuna persona > el oído para oir: Tú no escucbas, sólo oyes. $\| v$ prnl. 5 Mostrar <una persona > satisfacción de sí misma al hablar: Disfruta escuchándose.

\begin{tabular}{|c|c|c|c|}
\hline & $\begin{array}{l}\text { Relaciones } \\
\text { paradigmaticas de } \\
\text { significado: }\end{array}$ & $\begin{array}{l}\text { Rasgos } \\
\text { semanticos } \\
\text { distintivos } \\
\text { \& registro }\end{array}$ & $\begin{array}{l}\text { Información sobre la } \\
\text { combinatoria \& } \\
\text { contorno sintactico. } \\
\text { semantico }\end{array}$ \\
\hline 1 & poner atención & 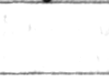 & $\begin{array}{l}\text { v.tr } \\
\text { Esq: <una persona> } \\
\text { [una cosa] }\end{array}$ \\
\hline 2 & dejarse influir por & & $\begin{array}{l}\text { vitr } \\
\text { Esq: <una persona> } \\
\text { lotra personal }\end{array}$ \\
\hline 3 & $\begin{array}{l}\text { oir. } \\
\text { percibir sonidos }\end{array}$ & $\mathrm{R}$ : amer & \\
\hline 4 & aplicar el oido & & $\begin{array}{l}\text { vintr. } \\
\text { Esq: <una persona> }\end{array}$ \\
\hline 5 & $\begin{array}{l}\text { mostrar satisfaccion al } \\
\text { hablar }\end{array}$ & & $\begin{array}{l}\text { v.pront. } \\
\text { Esq: <una persona> }\end{array}$ \\
\hline
\end{tabular}

Figura 4: escuchar de DSA (2006) y resumen esquemático de la información

16 En el ámbito de DaF son de especial interés los estudios lexicográficos que tratan los esquemas sintácticos (Dentschewa 2006; Gouws 1998; Schafroth 2002) y la inclusión de las combinaciones usuales o colocaciones (Köster / Neubauer 2002; Lehr 1998). 


\begin{tabular}{|c|c|c|c|c|}
\hline \multirow{2}{*}{ 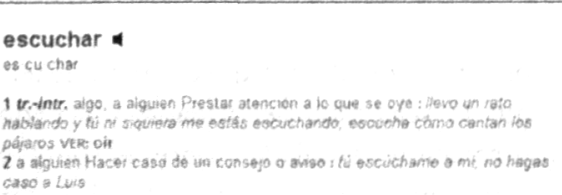 } & & $\begin{array}{l}\text { Relaciones } \\
\text { paradigmáticas } \\
\text { de significado }\end{array}$ & $\begin{array}{l}\text { Rasgos } \\
\text { semanticos } \\
\text { distintivos \& } \\
\text { registro }\end{array}$ & $\begin{array}{l}\text { Información sobre la } \\
\text { combinatoria \& } \\
\text { contorno sintáctico- } \\
\text { semantico }\end{array}$ \\
\hline & 12 & $\begin{array}{l}\text { prestar alencón a } \\
\text { lo que se oye } \\
\text { oir }\end{array}$ & & $\begin{array}{l}\text { vtr-intr } \\
\text { Esq: algo, a alguien }\end{array}$ \\
\hline \multirow[t]{2}{*}{ Evax, marce registrada por Larousse Editoriak, EUniversidad de Aicele } & 2 & $\begin{array}{l}\text { hacer caso de un } \\
\text { consejo } 0 \text { aviso }\end{array}$ & & Esq: a alguien \\
\hline & \multicolumn{4}{|c|}{ Esq esquerna } \\
\hline
\end{tabular}

Figura 5: escuchar de DA (formato online: cfr. diccionario.com: Portal vox-Larousse) y resumen esquemático de la información

Entre los diccionarios pedagógicos de Ele destaca además el "Diccionario de aprendizaje del español como lengua extranjera" (DAELE). "Es un diccionario monolingüe, dirigido a estudiantes de nivel avanzado, pensado principalmente para la producción" ( $c f r$. presentación del diccionario en la propia web), que ofrece en línea la información acerca de 363 verbos $^{17}$ del español y su combinatoria (figura 6). Es el único diccionario de Ele que aporta información sistemática sobre el potencial combinatorio mediante información acerca de las colocaciones más frecuentes y abundantes ejemplos:

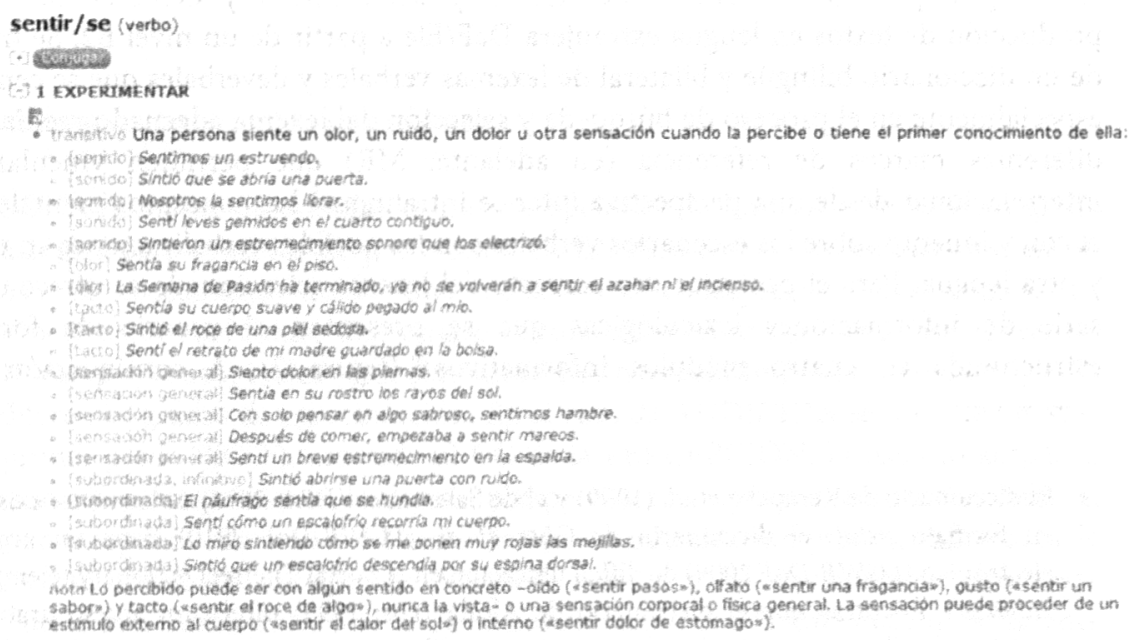

Figura 6: sentir de DAELE (secuencia)

Según el diccionario DaF y Ele en cuestión, la descripción de los mencionados parámetros puede ser más o menos sistemática y rigurosa, como queda expuesto en los ejemplos (figuras 3-6). A pesar de tratarse de diccionarios que pretenden aportar la información necesaria al usuario para procesos de redacción en $\mathrm{L} 2$, tal como queda expuesto en sendos prefacios, no gozan de un elevado índice de uso. Los

17 DAELE es un diccionario todavía en construcción que se desarrolla en el Institut Universitari de Lingǘstica Aplicada de la Universitat Pompeu Fabra (cfr. Battaner Arias 2008; Bernal / Renau 2010). 
motivos pueden ser, entre otros, la falta de accesibilidad al carecer en la mayoría de los casos de un libre acceso en línea. ${ }^{18}$

En resumen, cabe decir que tanto los procesos de búsqueda para lexemas desconocidos en la lengua extranjera como la selección del lexema adecuado y posteriormente su correcto uso con información detallada sobre el potencial combinatorio requieren del usuario una gran competencia lexicográfica. ${ }^{19}$ La gran oferta informativa a la que puede acceder el interesado mediante los portales lexicográficos no se aprovecha suficientemente, debido a un desconocimiento generalizado de la información que se encuentra detrás de cada portal. Además se puede afirmar que en general excede la capacidad de un estudiante de lengua extranjera. Surge, pues, la propuesta de un nuevo tipo de diccionario DICONALEonline que se presenta a continuación.

\section{DICONALE: buscar - seleccionar - usar}

DICONALE-online (diccionario conceptual del alemán y del español) pretende dar una respuesta a las lagunas arriba dibujadas y quiere ofrecer información lexicológica para una situación de uso muy determinada y definida: la libre producción de textos en lengua extranjera $\mathrm{DaF} /$ Ele a partir de un nivel B2. Se trata de un diccionario bilingüe y bilateral de lexemas verbales y deverbales que se centra especialmente en el proceso de búsqueda y selección del lexema adecuado mediante diferentes marcos de referencia (en adelante: MR) que permiten vincular $e$ interrelacionar desde una perspectiva inter- e intralingual los conceptos mentales y el conocimiento sobre los escenarios verbales con las posibles verbalizaciones en una y otra lengua. Para el posterior uso correcto del lexema seleccionado se ofrece una serie de informaciones lexicológicas que se presentan al usuario de forma estructurada en cuatro módulos informativos ${ }^{20}$ (figura 7). A continuación se

El diccionario de Kempcke et al. (1999) y el de Salamanca (DSA: 2006) únicamente existen en formato print, el diccionario de Götz et al. (LGWB-DaF 2010) tiene un acceso electrónico ( $L G W B-D a F 2009$ ) de difícil ubicación en el portal ThefreeDictionary (ejemplo: zuhören), mientras que el diccionario de Alcalá (DA: 1995) ofrece un acceso gratuito restringido. Por otra parte, los diccionarios con libre acceso como son p. ej. Pons-DaFonline (cfr. portal Pons) y Clave (portal SM) aportan escasa información a nivel sintagmático. Únicamente el diccionario DAELE ofrece mediante un acceso libre una amplia información especialmente dirigida a situaciones de producción, pero de momento no abarca más que una selección de algo menos de 400 verbos.

19 La información que nos aportan los FrameNet (Boas 2013; Boas / Dux 2013) tampoco no suele estar al alcance de los usuarios aquí enfocados.

20 Se ha confeccionado un modelo de descripción de 5 niveles mediante el cual se establecen las bases para la Macro- Medio- y Microestructura del diccionario a nivel inter- e intralingual. Para información más detallada sobre las bases teóricas y metodológicas de DICONALE remito a los siguientes estudios, elaborados en torno al proyecto: Meliss / Sánchez Hernández (2014), González Ribao / Meliss (2014), Egido Vicente / Fernández Méndez / Franco Barros (2014) y Fernández Méndez / González Ribao / Meliss (2014). 
describen los mecanismos de búsqueda y selección y los parámetros lexicológicos que se ofrecen para su uso:

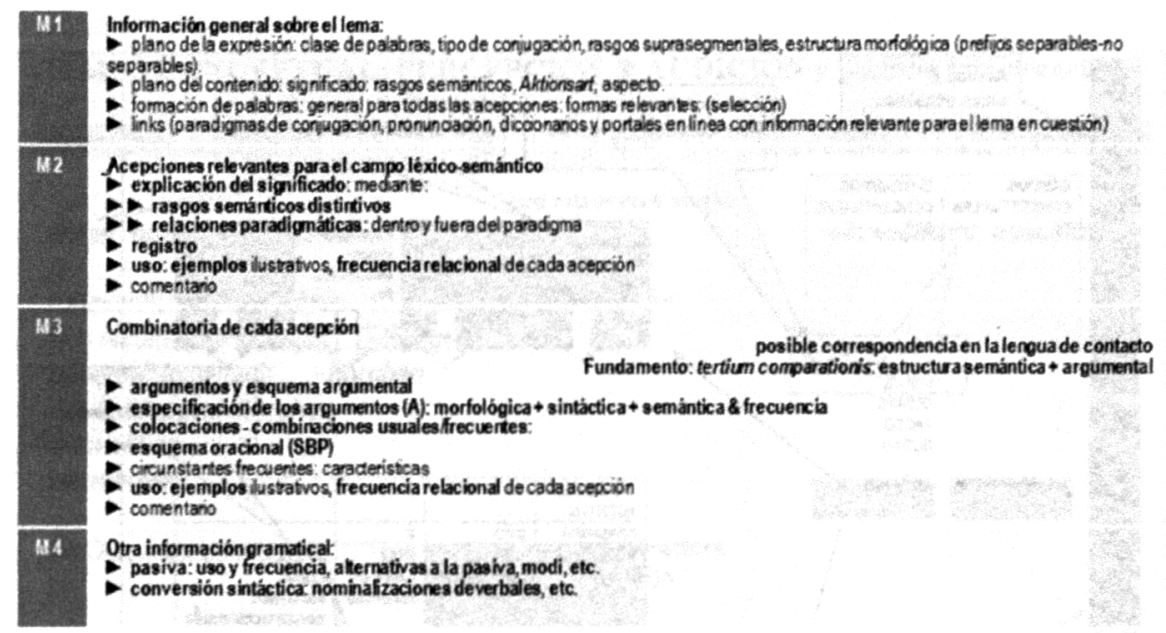

Figura 7: Los 4 módulos de descripción del modelo DICONALE

\subsection{Buscar lo desconocido y seleccionar}

DICONALE-online quiere ofrecer nuevas opciones de búsqueda en la lexicografía pedagógica para procesos de producción en L2. Para ello centra su atención en un acceso al diccionario desde una perspectiva conceptual-onomasiológica. La Macroestructura del futuro diccionario se constituye en una primera fase de trabajo de 10 campos conceptuales (CC) con sus respectivos subcampos conceptuales (SCC) de diferentes grados de especificación. En el caso de AUDICIÓN se trata de un SCC de primer grado $\left(\mathrm{SCC}^{1}\right)$, asociado al $\mathrm{CC}$ de la PERCEPCIÓN. El usuario accede al diccionario principalmente desde una perspectiva conceptual que puede realizar en alemán o español como L1 o L2 según la opción inicialmente predeterminada. Se le ofrece un detallado sistema de búsqueda guiada $\mathrm{y}$ oferta multiselección para encontrar una forma léxica adecuada en combinación con la información pertinente para el uso correcto en relación a los propósitos comunicativos. Dos marcos de referencia (en adelante: MR) que a la vez sirven de tertium comparationis entre ambas lenguas forman las guías principales a las que se añaden en pasos sucesivos otras opciones para afinar la selección de una u otra variante léxica. El primer MR abarca las referencias conceptuales con diferente grado de especificación (MR1) mientras que el segundo se aproxima a la descripción del escenario verbal indicando los diferentes roles semánticos involucrados (MR2). Ambos marcos de referencia forman la base para la principal opción de acceso al diccionario (acceso 1) que a su vez permite diferentes especificaciones (la-c). A mayores se ofrece también una búsqueda clásica por orden alfabético de los lemas (acceso 2), cuya opción no lleva, sin embargo, ni a equivalentes de traducción ni a una perspectiva semasiológica 
tradicional, sino reconduce al usuario a una información guiada por los marcos de referencia arriba indicados. En la figura 8 se visualizan los diferentes modos de búsqueda y los accesos iniciales que a continuación se presentan detalladamente:

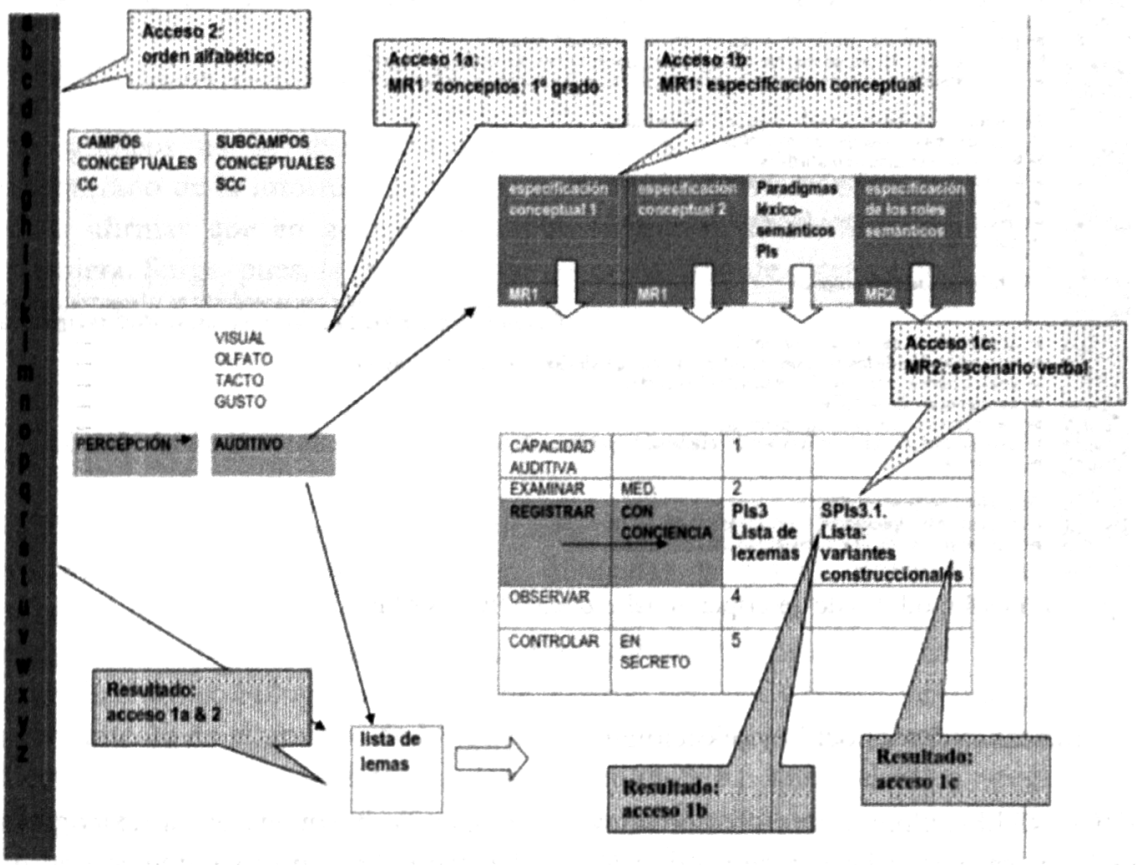

Figura 8: Diferentes posibilidades de acceso inicial a DICONALE-online para el proceso de búsqueda de formas de expresión ${ }^{21}$

3.1.1. El marco de referencia 1: En el caso de querer conocer en la L2 todas las posibles lexicalizaciones para los conceptos PERCEPCIÓN \& AUDICIÓN, el interesado selecciona mediante la estructura inicial del diccionario el CC PERCEPCIÓN y a continuación, en un menú desplegable se le ofrece la posibilidad de especificar su consulta en diferentes grados. La especificación conceptual del primer grado llevaría a los SCC AUDICIÓN / VISUAL / TACTIL / OLFATO / GUSTO. El resultado de esta búsqueda por conceptos de primer grado son listados de lemas que lexicalizan en ambas lenguas los conceptos básicos buscados. Estos listados incluyen en ambas lenguas lemas verbales simples, afijados y también formas léxicas complejas y otras construcciones frecuentes así como una selección de nombres, adjetivos y adverbios deverbales, como puede verse a modo de ejemplo en la figura 9 para las posibles lexicalizaciones de AUDICIÓN. Estos listados resultan únicamente útiles si posteriormente se usan los enlaces creados en cada

21 Las figuras 8-13 no reproducen la interfaz de la aplicación web del futuro Diccionario DICONALE. 
lema para acceder a la información lexicológica relevante que permita asociar mediante la especificación conceptual una acepción a un paradigma léxicosemántico (cfr. apartado 3.1.3. y figura 12):

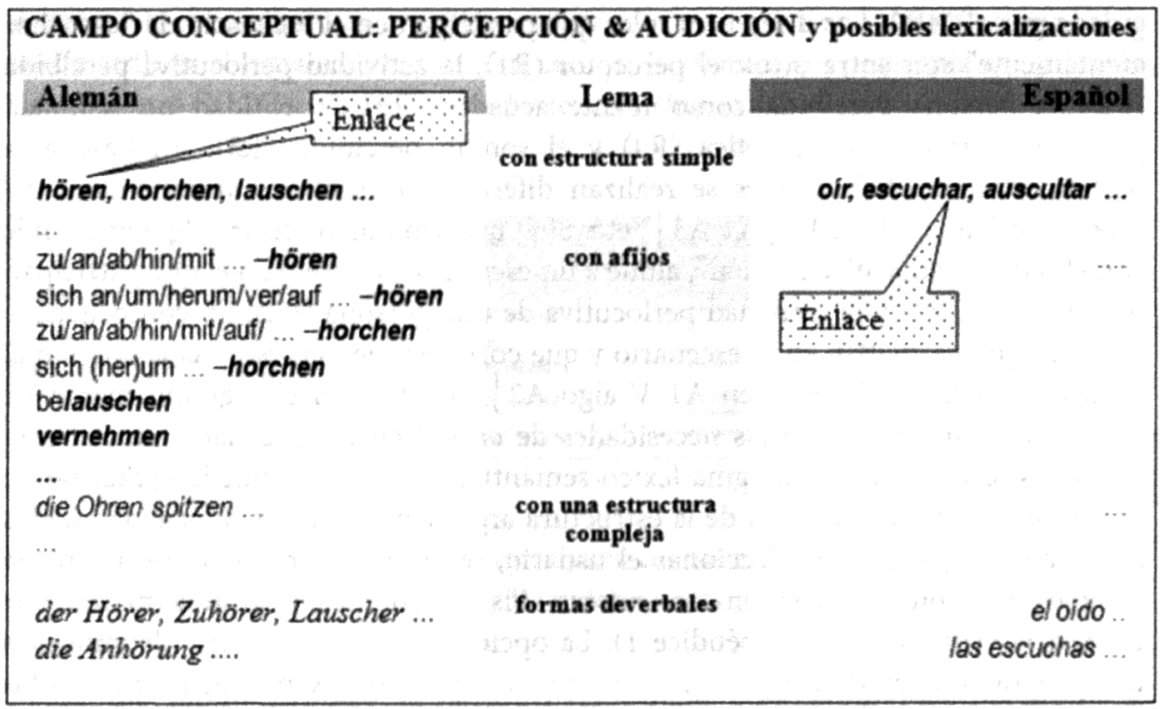

Figura 9: Listado de lemas: posibles lexicalizaciones en alemán y español para AUDICIÓN

Si por otra parte, el usuario prosigue con una búsqueda por conceptos cada vez más específicos, es conducido directamente a los diferentes paradigmas léxicosemánticos. Para ello es necesario ofrecerle un marco de referencias conceptuales muy específicas. Así, p. ej. la selección de los conceptos PERCEPCION \& AUDICIÓN \& INTENCIONALIDAD \& ATENCIÓN conduce a un Paradigma léxico-semántico (Pls3) determinado y se diferencia de otros posibles Pls vinculados igualmente al concepto PERCEPCIÓN AUDITIVA por corresponder a un MR1 diferente. Como resultado de esta búsqueda recibimos los recursos lingüísticos de ambas lenguas mediante los cuales se verbalizan los conceptos específicos en cuestión y que corresponden al mismo MR1. La figura 10 (cfr. apéndice 1) refleja de forma ejemplar el resultado de la búsqueda realizada mediante la especificación conceptual correspondiente al MR1 (alemán: (sich) anhören1, zuhören1, hören2, horchen1, lauschen1; español: escuchar $2 y$ oir 2). Una selección de ejemplos ilustra el significado de cada lexema en cuestión. Por consiguiente, no se incluyen en este paradigma lexemas como al. abhören, abhorchen, belauschen, etc. y esp.: auscultar, etc., ya que no corresponden en ninguna de sus posibles acepciones al MR1 establecido para el Pls3.

3.1.2. La búsqueda mediante el marco de referencia 2: Para los fines aquí relevantes es preciso delimitar todavía más la búsqueda iniciada mediante el MR1. El usuario parte con sus necesidades comunicativas no solamente de unos conceptos determinados para los que busca posibilidades de expresión. Parte también de un escenario concreto que quiere verbalizar en la L2. Mediante la descripción de los 
escenarios se especifica la búsqueda y selección de posibles lexemas para las necesidades comunicativas concretas. En base al paradigma Pls3 que corresponde al marco de referencias conceptuales PERCEPCIÓN \& AUDICIÓN \& ATENCIÓN \& INTENCIONALIDAD, se ejemplifica a continuación esta búsqueda y selección guiada por el MR2. Los diferentes roles que participan en el escenario de "escuchar atentamente" son entre otros: el perceptor (R1), la actividad perlocutiva percibida (R2), la persona percibida como fuente acústica (R3), la entidad no animada percibida como fuente acústica (R4) y el sonido percibido (R5). En base a la participación de dichos roles se realizan diferentes estructuras argumentales del lexema verbal: $|\mathrm{A} 1 \mathrm{~A} 2|,|\mathrm{A} 1 \mathrm{~A} 3|$, etc. Si el usuario quiere decir "alguien atiende con el oído a lo que dice alguien", alude a un escenario concreto en el que participan un perceptor (R1) y la actividad perlocutiva de una persona (R2). En consecuencia, los roles que participan en el escenario y que corresponden en este caso concreto al esquema argumental |alguien A1 $V$ algo A2 constituyen el segundo marco de referencias comunes. Para las necesidades de output en L2 el usuario únicamente busca los lexemas del paradigma léxico-semántico con los que pueda verbalizar su escenario ideado. En función de la estructura argumental (cantidad y calidad de los argumentos) que puede seleccionar el usuario, se le ofrece un conjunto u otro de posibles variantes léxicas en un mismo Pls al compartir ambos marcos de referencia ${ }^{22}$ (figura 11, cfr. apéndice 1). La opción de especificación y delimitación que proporciona el MR2 es relevante, ya que no todos los lexemas de un mismo Pls comparten los mismos escenarios. Así, por ejemplo, podemos observar que según la información empírica sobre las posibles coocurrencias de lauschen y zuhören (CCDB: Belica 2011), el verbo lauschen aparece frecuentemente vinculado al escenario: "alguien (R1: perceptor) sigue atentamente con el oído un sonido (R5: sonido: Klang, Gesang, Geräusch...)", mientras que zuhören prioriza otros escenarios (p. ej.: R1: perceptor \& R3: fuente acústica animada: Leute ...). Una vez seleccionada la variante léxica que más se ajuste a las necesidades comunicativas, el usuario puede obtener en un siguiente paso información lexicológica detallada correspondiente a los cuatro módulos de descripción (cfr. figura 8).

Aunque la perspectiva conceptual de DICONALE favorece el acceso desde la L2, se permite también el acceso desde el español y el alemán como L1. En cualquier caso la búsqueda y selección siempre pasa por ambos marcos de referencias y no se ofrecen posibles equivalencias en la lengua de contacto (con la opción de obtener la información léxica acerca de los diferentes módulos de descripción) hasta no haber desambiguado al máximo a nivel semántico y sintáctico mediante ambos marcos de referencia (figura 12$)^{23}$

Cfr. Engelberg (2010, 2014), Engelberg et al. (2011), Engelberg et al. (eds., 2012). El volumen de Engelberg / Meliss / Proost / Winkler (eds., 2014) reúne algunos estudios que analizan las estructuras argumentales referentes a algunos paradigmas verbales en contraste (alemán-español).

23 Para la discusión teórica sobre diferentes aspectos generales de la lexicografia bilingüe remito a los siguientes estudios: Fuentes Morán / Model (2009), Haensch / Omeñaca (2004²), Hausmann (1991), Hausmann / Werner (1991), Korhonen (2001), Meyer / 
3.1.3. Búsqueda por lema: $\mathrm{Si}$ el interesado ya conoce alguna posible lexicalización para sus necesidades comunicativas, puede realizar en la lengua que decida una búsqueda mediante un lema por orden alfabético (acceso 2: figura 8). Al contrario de lo habitual, el resultado es en primer lugar un cuadro general del CC al que se le puede atribuir el lema en cuestión. En caso de homonimia o polisemia se indicarán los diferentes CCCC a los que pueda pertenecer (figura 12).

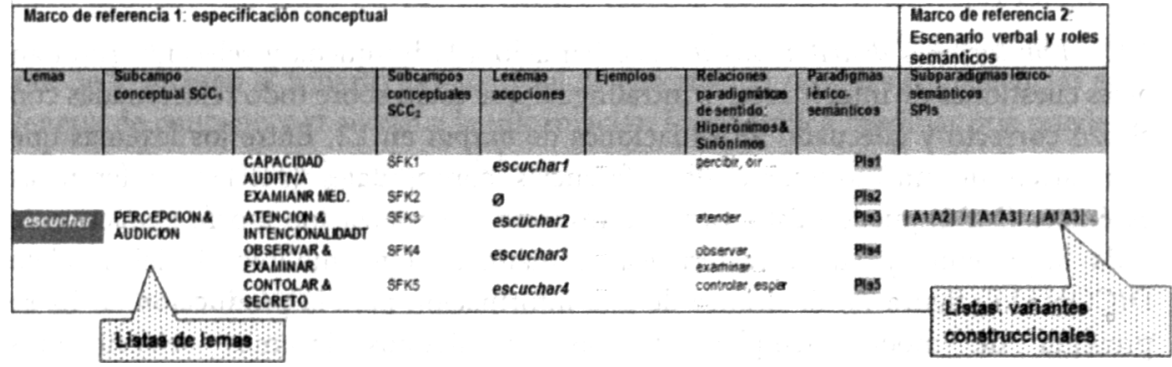

Figura 12: Búsqueda por lema: escuchar: vinculación con los marcos de referencias MR1 y MR2 y los correspondientes listas de lexemas y listas de variantes construccionales correspondientes a cada SPls

De ese modo escuchar se atribuye al campo conceptual PERCEPCIÓN \& AUDICIÓN y se le abre al usuario la posibilidad de visualizar todos los elementos léxicos asociados a este CC (lista de lemas: figura 9). Por otra parte, mediante una especificación conceptual y con la ayuda de un ejemplo ilustrativo se asocian las diferentes acepciones de escuchar indicadas con un índice numérico (1-4) a los diferentes (sub)paradigmas léxico-semánticos correspondientes al CC AUDICIÓN. A partir de esta búsqueda el interesado llega a relacionar cada acepción con uno $\mathrm{u}$ otro Pls y accede al listado de los lexemas vinculados al paradigma en cuestión (figuras 10 y $11 ;$ cfr. apéndice 1 ).

3.1.4. Otros factores distintivos para la selección entre la variedad: En base a una descripción sistemática de los diferentes parámetros a nivel del contenido y la forma, tal como lo prevén los módulos de descripción lexicológica 2 y 3 (figura 7), se pueden plasmar las divergencias entre los lexemas que comparten los mismos marcos de referencia en un mismo (sub)paradigma léxico-semántico a nivel inter- $\mathrm{e}$ intralingual. Es un requerimiento imprescindible visualizar estas divergencias en su conjunto para facilitar al usuario la tarea de selección entre la variedad en situaciones de output en L2. De modo ejemplar se presenta a continuación la información correspondiente al escenario "alguien escucha lo que dice alguien" materializado en la estructura argumental $\mid$ A1 A2 $\mid$ vinculado al Pls3 del SCC AUDICIÓN. Para la selección de una de las posibilidades entre la variedad que se nos ofrece, es preciso disponer de elementos distintivos. Junto a los rasgos 
semánticos distintivos [genau] y [konzentriert] son relevantes las informaciones acerca de las posibilidades combinatorias más frecuentes y sus delimitaciones distributivas. Así, el verbo anhören puede combinar con "Beschimpfung" y "Beschwerde" (= escuchar insultos, quejas) - posibilidad que no se documentó para los demás verbos del Pls3 con la estructura argumental |A1 A2 |. Para estos casos es de especial interés tener la opción de acceder a la información sistematizada en contraste sobre las colocaciones más frecuentes de todos los lexemas de un mismo Pls (figura 12).

3.1.5. Limitaciones distributivas de uso: Paralelo a la búsqueda y selección resultan otras cuestiones de interés inter-e intralingual que están sobre todo relacionadas con el uso correcto y adecuado en situaciones de output en L2. Entre los lexemas que comparten un mismo marco de referencias conceptuales (MR1) y referencias escénicos (MR2) puede existir variedad respecto a la realización morfo-sintáctica de cada argumento y sus características semánticas (cfr. módulo 3 del modelo DICONALE: figura 7). El alcance de esta información para la producción en L2 se presenta de modo ejemplar en base a algunos lexemas seleccionados correspondientes al MR1 y MR2 agrupados en el SPls3 del SCC AUDICIÓN (figura 13, cfr. apéndice 2). El análisis demuestra, por ejemplo, que algunos lexemas verbales del SPls3al realizan el argumento A2 como complemento directo (akk): (sich) anhören y hören (ejemplos: $2 \mathrm{dt}-3 \mathrm{dt}$ ) o como complemento indirecto (dat): zuhören, lauschen y horchen (ejemplos: $1_{\mathrm{dt}}, 4 \mathrm{a}_{\mathrm{dt}}, 5 \mathrm{a}_{\mathrm{dt}}$ ). En lauschen 1, hören2, horchen 1 existen además alternancias entre diferentes formas de realización morfosintáctica del argumento A2 (ejemplos: $4 \mathrm{a}_{\mathrm{dt}}-4 \mathrm{~b}_{\mathrm{dt}}, 5 \mathrm{adt}_{\mathrm{dt}}-5 \mathrm{~b}_{\mathrm{dt}}$ ). La información sobre posibles realizaciones oracionales de los argumento es igualmente de interés y ayuda a la variación estilística a la hora de producir un texto en L2:

(1 $\left.1_{\mathrm{dt}}\right)$ Mit großem Interesse hatten die Pflegekräfte [...], dem Vortrag zugehört [...]. (M03/FEB.11701 Mannh. Morgen, 22.02.2003).

(2dt) Sie hören sich die Probleme an, die den Kindern auf den Nägeln brennen [...]. (R97/SEP.72386 Frankf. Rundschau, 15.09.1997, S. 4).

(3dt) Sie hörte die Aussagen der verängstigten Kinder. (RHZ05/JUN.16629 RZ, 15.06.2005).

(4adt) Man setzt sich, horcht der Feldpredigt und geniesst die Sonne. (A00/JUN.38103 St. Galler Tagblatt, 02.06.2000)

(4bdt) Er horchte auf die Worte der Reisenden, die von Bord gingen, und wenn sie deutsch sprachen, redete er sie an. (P97/MAR.11400 Die Presse, 22.03.1997).

(5adt) Rund ein Drittel der Steinacher Ortsbürger lauschten den Worten des Präsidenten, als dieser auf das vergangene Jahr zurückblickte. (A13/APR.06663 St. Galler Tagblatt, 17.04.2013, S. 34).

(5bdt) Gemeinsam mit Lehrerin Ilka Peter hatten sich zehn Kinder aus den Klassen Zwei, Drei und Vier versammelt und lauschten auf die Erzählung „Weihnachten im Möwenweg“" von Kirstin Boie. (M08/DEZ.95513 Mannh. Morgen, 08.12.2008, S. 19).

Los diccionarios monolingües pedagógicos consultados no aportan información sobre lexemas en contraste, a pesar de ser una información muy pertinente, no 
solamente en situaciones de producción en lengua extranjera (Wolf 2001). Toda esta información detallada es útil a la hora de redactar un texto en la lengua extranjera y le ofrece al usuario un amplio abanico de posibilidades de expresión entre las cuales puede seleccionar teniendo en cuenta las peculiaridades de uso de cada uno de los lexemas. La información sobre las posibilidades de construcción en pasiva y sobre posibles productos de formación de palabras ( $c f r$. módulo 4 del modelo DICONALE: figura 7) completan la información detallada que debe estar a disposición del usuario para producir un texto en L2. Se dedica especial atención a la visibilidad de las divergencias a nivel del contenido, formal y funcional entre ambas lenguas, por lo que se le ofrecen al usuario en este nivel de descripción posibles equivalencias en la lengua de contacto y el acceso a la información lexicográfica modular que puede ser consultada según las necesidades del usuario (figura 13, cfr. apéndice 2).

\section{Reflexiones finales}

A lo largo de este trabajo se ha demostrado que los diccionarios mono- y bilingües de aprendizaje en contextos de DaF y Ele no contemplan lo suficiente el proceso de buscar, seleccionar y usar para la producción en la lengua extranjera. En respuesta a las deficiencias en la lexicografía bilingüe y monolingüe de aprendizaje de DaF y Ele se han elaborado algunas propuestas en el marco de la creación de DICONALE que pretenden ofrecer soluciones a la falta de recursos lexicográficos adecuados para la situación aquí en cuestión. Dos marcos de referencia constituyen por un lado la base para la agrupación de los lexemas en paradigmas léxico-semánticos y por otro forman el tertium comparationis entre ambas lenguas en contraste. La descripción del significado mediante la combinación de diferentes parámetros (rasgos semánticos y relaciones paradigmáticas de sentido, etc.) y la descripción detallada del potencial combinatorio tomando como punto de partida las estructuras argumentales, son las bases de los módulos centrales de descripción. La bilateralidad del diccionario bilingüe, en el que la lengua meta L2 puede ser alemán o español, y la complejidad de las interrelaciones léxicas justifica entre muchas otras razones como pueden ser la comodidad, rapidez e interrelación de información el formato online. ${ }^{24}$ Una consulta modular según las necesidades del usuario enlaza a su vez con una base metodológica empírica que comprende información tanto de los corpus de referencia del español y alemán como de la web como corpus.

Para más información sobre diferentes aspectos actuales en torno a la lexicografía online y la lexicografía computacional remito a los siguientes estudios: Aguilar Escobar (2009), Chuchuy / Moreno (2002), Haß / Schmitz (2010), Haß (2005), Klein / Geyken (2010), Klosa (2008, 2009), Mann (2010), Müller-Spitzer / Engelberg (2011, 2013), Pöll (2010), Sanmarco Bande (2008), Storrer (2010), Tarp (2012) y Torres del Rey (2009). 


\section{Referencias bibliográficas}

Diccionarios, portales lexicográficos, corpora

Bosque, Ignacio (2004): Redes. Diccionario combinatorio del español contemporáneo. Madrid: Ediciones SM.

CanooNet: Portal: Deutsche Wörterbücher und Grammatik. Disponible en <http://www. canoo.net/> [Consulta: 01/03/2014].

Casares, Julio (1942/2011): Diccionario ideológico de la lengua española. Barcelona: G. Gili.

CCDB: Belica, Cyril (2011): Kookkurrenzdatenbank V3.3. Eine korpuslinguistische Denk- und Experimentierplattform. Institut für Deutsche Sprache: Mannheim. Disponible en $<$ http://corpora.ids-mannheim.de/ccdb/> [Consulta: 01/03/2014].

CLAVE-online: Diccionario de uso del español actual. Portal SM-Diccionarios. Disponible en $<$ http://clave.smdiccionarios.com/app.php > [Consulta: 01/03/2014].

COSMAS: Corpus Search Management and AnalysisSystem. Institut für Deutsche Sprache: Mannheim. Disponible en <http://www.ids-mannheim.de/cosmas2/uebersicht.html> [Consulta: 01/03/2014].

CREA: Corpus de referencia del español actual. Real Academia Española. Disponible en $<$ http://corpus.rae.es/creanet.html> [Consulta: 01/03/2014].

Cuervo, Rufino J. (1953/1998): Diccionario de construcción y régimen de la lengua castellana. Instituto Caro y Cuervo (ed.): Barcelona: Herder.

DA: Moreno Fernández, Francisco (coord.) (1995/2010²): Diccionario para la Enseñanza de la Lengua Española (Diccionario de Alcalá). Barcelona: Vox.

DAELE: Diccionario de aprendizaje del español como lengua extranjera: $<$ http://www.iula.upf.edu/rec/daele/> [Consulta: 01/03/2014].

DCVVEA: Diccionario contrastivo de valencias verbales: español-alemán. <http://gramatica.usc.es/proxectos/valencia/diccionario/> [Consulta: 01/03/ 2014].

DEA: Seco, Manuel et al. (1999): Diccionario del español actual. 2 tomos. Madrid: Aguilar.

DEE: Maldonado, Concepción (coord.) (2002): Diccionario de Español para Extranjeros. Madrid: SM.

DeReKo: Deutsches Referenzkorpus des IDS-Mannheim (Institut für Deutsche Sprache Mannheim).

COSMAS II: (Corpus Search, Management and Analysis System). Disponible en $<$ https://cosmas2.ids-mannheim.de/cosmas2-web/> [Consulta: 01/03/2014].

Diccionarios.com: Portal Vox \& Larousse <http://www.diccionario.com/> [Consulta: 01/03/2014].

DiCE: Diccionario de colocaciones del español: <http://www.dicesp.com/paginas $>$ [Consulta: 01/03/2014].

DICONALE: Diccionario conceptual del alemán y del español. Konzeptuell orientiertes Wörterbuch: Deutsch-Spanisch. USC. Disponible en <http://www.usc.es/gl/proxectos/ diconale/aleman/> [Consulta: 01/03/2014].

Dornseiff, Franz / Wiegand, Herbert E. / Quasthoff, Uwe $\left(2004^{8}\right)$ : Der deutsche Wortschatz nach Sachgruppen. Berlin: de Gruyter (en papel / electrónico).

DRAE (1992 $\left.21 / 2001^{22}\right)$ : Diccionario de la Lengua Española. Real Academia Española, Madrid: Espasa Libros.

DRAEe: Diccionario de la Real Academia Española. Acceso electrónico: $<$ http://buscon.rae.es/draeI/> [Consulta: 01/03/2014].

DSA: Gutiérrez Cuadrado, Juan / Pascual Rodríguez, J. Antonio (coord.) (2006): Diccionario de Salamanca - Español para extranjeros. Madrid: Santillana.

Duden (20109): Stilwörterbuch. Mannheim: Dudenverlag.

Duden-online Portal: <http://www.duden.de/> [Consulta: 01/03/2014]. 
DWDS: Digitales Wörterbuch der deutschen Sprache. Disponible en <http://www.dwds.de/> [Consulta: 01/03/2014].

elexiko: Online-Wörterbuch der deutschen Gegenwartssprache. IDS-Mannheim <http://www.owid.de/wb/elexiko/start.html> [Consulta: 01/03/2014].

Kempcke, Günter et al. (1999): Wörterbuch Deutsch als Fremdsprache. Berlin: de Gruyter.

$K V$-online: Online Nachschlagewerk zu Kommunikationsverben. IDS-Mannheim. Disponible en <http://www.owid.de/docs/komvb/start.jsp > [Consulta: 03/01/2014].

LEO (Portal lexicográfico multilingüe para consultas bilinguales) <http://www. leo.org/> [Consulta: 01/03/2014].

LGWBe-DaF: Götz, Dieter / Haensch, Günter / Wellmann, Hans (2007/2010): Langenscheidts $e$-Großwörterbuch Deutsch als Fremdsprache. Berlin, München: Langenscheidt.

MM: Moliner, María (1970/2007): Diccionario de uso del español. Madrid: Gredos.

OpenThesaurus: Freies deutsches Wörterbuch für Synonyme. Disponible en $<$ http://www.openthesaurus.de/> [Consulta: 01/03/2014].

OWID: Online-Wortschatz-Informationssystem Deutsch. IDS Mannheim. Disponible en $<$ http://www.owid.de/> [Consulta: 01/03/2014].

Pons: Das Sprachenportal. Disponible en <http://de.pons.eu/> [Consulta: 01/03/2014].

Pons-DaF (2006): Großwörterbuch Deutsch als Fremdsprache. Stuttgart: Klett (en papel / electrónico)

Pons-DaFe: Deutsch als Fremdsprache online: <http://de.pons.eu/> [Consulta: 01/03/2014].

Quasthoff, Uwe (2011): Wörterbuch der Kollokationen im Deutschen. Berlin: de Gruyter.

TheFreeDictionary: <http://de.thefreedictionary.com/> [Consulta: 01/03/2014].

VALBU: Schumacher, Helmut et al. (2004): Valenzwörterbuch deutscher Verben. Tübingen: Narr [Consulta: 01/03/2014].

VALBU-e: E-Valbu: Valenzwörterbuch online. IDS-Mannheim. Disponible en <http:// hypermedia.ids-mannheim.de/evalbu/index.html> [Consulta: 01/03/ 2014].

Wehrle, Hugo / Eggers, Hans (1961/1993 $\left.{ }^{17}\right)$ : Deutscher Wortschatz: ein Wegweiser zum treffenden Ausdruck. Stuttgart: Klett.

WordReference: Diccionario de Español, Inglés Francés y Portugués. Disponible en $<$ http://www.wordreference.com/es/> [Consulta: 01/03/2014].

Wörterbuch für Synonyme: online <www.synonym.de $>$ [Consulta: 01/03/2014].

Wortschatz Universität Leipzig: Portal <http://wortschatz.uni-leipzig.de/> [Consulta: 01/03/2014].

Woxikon: Online-Wörterbuch: Wörterbuch und Übersetzung: <www.woxikon. de> [Consulta: 01/03/2014].

Estudios

Aguilar Escobar, Gonzalo (2009): Los diccionarios electrónicos del español. Madrid: Arco Libros.

Alonso Ramos, Margarita (2004): «Elaboración del Diccionario de colocaciones del español y sus aplicaciones", en Paz Battaner Arias / Janet A. de Cesaris, eds., De Lexicografía. Actes del I Simposium Internacional de Lexicografía. Barcelona: IULA y Edicions Petició, 149-162.

- (2008): «Papel de los diccionarios de colocaciones en la enseñanza de español como L2», en Elisende Bernal / Janet A. de Cesaris, eds., Proceedings of the Xiii Euralex International Congress. Barcelona: Documenta Universitaria, Série Activitats, 1215-1230.

Battanner Arias, $\mathrm{Paz}$ (2008): «Sobre las construcciones pronominales y su tratamiento en algunos diccionarios monolingües de cuatro lenguas románicas", en Elisende Bernal / Janet A. de Cesaris, eds., Proceedings of the Xiii Euralex International Congress. Barcelona: Documenta Universitaria, Série Activitats, 495-504. 
Bernal, Elisenda / Renau, Irene (2010): « ¿Lo que necesitan es lo que encuentran? Reflexiones a propósito de la representación de los verbos en los diccionarios de aprendizaje del español", en Aanne Dykstra / Tanneke Sschoonheim, eds., Proceedings of the XIV Euralex International Congress 2010. Ljouwert: Fryske akademy - Afûk, 484-496.

Boas, Hans (2013): «Wieviel Wissen steckt in Wörterbüchern? Eine Frame-semantische Perspektive", Zeitschrift für Angewandte Linguistik, 57, 75-97.

Boas, Hans / Dux, Ryan (2013): «Semantic frames for foreign-language education: Towards a German frame-based dictionary", Veridas On-Line, 1/2013, 81-100.

Campos Souto, Mar / González González, E. Ma / Pérez Pascual, J. I. (eds.) (2008): La lexicografía bilingüe y didáctica: ayer y hoy. Anexos Revista de Lexicografia, 8. La Coruña: Universidade da Coruña.

Chuchuy, Claudio / Moreno, Antonio (2002): «Diccionarios españoles en formato electrónico», en $\mathrm{M}^{\mathrm{a}}$ Teresa Fuentes Morán / Reinhold Werner, eds., Lexicografias iberrománicas: problemas, propuestas y proyectos. Frankfurt: Vervuert, 89-108.

Dentschewa, Emilia (2006): «DaF-Wörterbücher im Vergleich: Ein Plädoyer für "Strukturformeln“", en Ana Dimova et al., eds., Zweisprachige Lexikographie und Deutsch als Fremdsprache. Hildesheim: Olms, 113-128.

Domínguez Vázquez, Ma José / Lübke, Barbara / Meliss, Meike / Paredes, Gemma / Poulsen, Pia / Vázquez, Victoria (2008a): «Presentación del Diccionario contrastivo de valencias verbales: español-alemán. Primera Parte: características generales del proyectom, en Mar Campos Souto et al., eds., La lexicografía bilingüe y didáctica: ayer y hoy. Anexos Revista de Lexicografia, 8. La Coruña: Universidade da Coruña, 51-62.

- (2008b): «Presentación del Diccionario contrastivo de valencias verbales: español-alemán. Segunda Parte: problemas descriptivos particulares", en Mar Campos Souto et al., eds., La lexicografía bilingüe y didáctica: ayer y hoy. Anexos Revista de Lexicografía, 8. La Coruña: Universidade da Coruña, 63-74.

Egido Vicente, María / Fernández Méndez, Manuel / Franco Barros, Mario (2014): «Propuesta para un nuevo tipo de diccionario pedagógico para DaF y Elem, en João Paulo Silvestre et al., eds., Dicionários que não existem. Lisboa: Editorial (en prensa).

Engel, Urich (2004): Deutsche Grammatik. - Neubearbeitung. München: iudicium.

Engelberg, Stefan (2010): «Die lexikographische Behandlung von Argumentstrukturvarianten in Valenz- und Lernerwörterbüchern", en Klaus Fischer / Eilika Fobbe / Stefan Schierholz, eds., Valenz und Deutsch als Fremdsprache. Frankfurt: Peter Lang, 113-141.

- (2014): "The argument structure of psych-verbs: A quantitative corpus study on cognitive entrenchment", en Hans Boas / Alexander Ziem, eds., Constructional approaches to argument structure in German. Boston, Berlin: de Gruyter Mouton (en prensa).

Engelberg, Stefan / Lemnitzer, Lothar $\left(2001,2009^{4}\right)$ : Lexikographie und Wörterbuchbenutzung. Tübingen: Stauffenburg.

Engelberg, Stefan / König, Svenja / Proost, Kristel / Winkler, Edeltraud (2011): "Argumentstrukturmuster als Konstruktionen? Identität - Verwandtschaft Idiosynkrasien", en Stefan Engelberg / Anke Holler / Kristel Proost, eds., Sprachliches Wissen zwischen Lexikon und Grammatik. Berlin: de Gruyter, 71-112.

Engelberg, Stefan / Koplenig, Alexander / Proost, Kristel / Winkler, Edeltraud (2012): «Argument structures and text genre: cross-corpus evaluation of the distributional characteristics of argument structure realizations", Lexicographica, 28, 13-48.

Engelberg, Stefan / Meliss, Meike / Proost, Kristel / Winkler, Edeltraud (eds.) (2014): Argumentstruktur - Valenz - Konstruktionen. Tübingen: Narr, (en prensa)

Fernández Méndez, Manuel / González Ribao, Vanessa / Meliss, Meike (2014): «DICONALEonline: propuesta para un nuevo tipo de diccionario bilingüe alemán-español como lenguas extranjeras", en Meike Meliss / $\mathrm{M}^{\mathrm{a}}$ Dolores Sánchez Palomino / $\mathrm{M}^{\mathrm{a}}$ Teresa Sanmarco 
Bande, eds., A lexicografía das linguas románicas: Estado da cuestión. München: Iudicium (en prensa).

Fuentes Morán, $M^{a}$ Teresa (1997): Gramática en la lexicografía bilingüe. Morfología y sintaxis en diccionarios español-alemán desde el punto de vista del germanohablante. Tübingen: Niemeyer.

Fuentes Morán, Ma Teresa / Werner, Reinhold (eds.) (2002): Lexicografías iberrománicas: problemas, propuestas y proyectos. Frankfurt: Vervuert.

Fuentes Morán, Ma Teresa / Modal, Benedikt (eds.) (2009): Investigaciones sobre lexicografía bilingüe. Granada: Tragacanto.

González Ribao, Vanessa (2014): «Sobre algunos conflictos en la 'pre'-lexicografía: la selección de corpus para la elaboración de un diccionario conceptual alemán-español", en $\mathrm{M}^{\mathrm{a}}$ José Domínguez Vázquez / Xavier Gómez Guinovart / Carlos Valcárcel Riveiro, eds., Lexicografía de las lenguas románicas. II. Aproximaciones a la lexicografía moderna y contrastiva. Berlin/New York: de Gruyter, 223-269.

González Ribao, Vanessa / Meliss, Meike (2014): "Vorschläge zur Ausarbeitung eines onomasiologisch-konzeptuell orientierten Produktionswörterbuches im zweisprachigen Lernerkontext: Deutsch-Spanisch", en José A. Calañas Continente et al., eds., Wörterbücher des Deutschen. Frankfurt: Peter Lang (en prensa).

González Ribao, Vanessa / Proost, Kristel (2014): «El campo léxico al servicio de la lexicografía: Un análisis contrastivo en torno a algunos subcampos de los verbos de comunicación en alemán y español», en $\mathrm{M}^{\mathrm{a}}$ José Domínguez Vázquez / Xavier Gómez Guinovart / Carlos Valcárcel Riveiro, eds., Lexicografia de las lenguas románicas II. Aproximaciones a la lexicografia moderna y contrastiva. Berlin/New York: de Gruyter, 223-245.

Gouws, Rufus H. (1998): «Das System der sogenannten Strukturformeln in LANGENSCHEIDTS GROßWÖRTERBUCH DEUTSCH ALS FREMDSPRACHE: eine kritische Übersicht", en Herbert E. Wiegand, ed., Perspektiven der pädagogischen Lexikographie des Deutschen. Untersuchungen anhand von "Langenscheidts Großwörterbuch Deutsch als Fremdsprache". Tübingen: Niemeyer, 63-76.

Haensch, Günther / Omeñaca, Carlos $\left(1997,2004^{2}\right)$ : Los diccionarios del español en el siglo XXI. Salamanca: Ediciones Universidad de Salamanca.

Harras, Gisela / Winkler, Edeltraud et al. (2004): Handwörterbuch deutscher Kommunikationsverben. Teil 1: Wörterbuch. Berlin: de Gruyter.

Harras, Gisela / Proost, Kristel / Winkler, Edeltraud (2007): Handbuch deutscher Kommunikationsverben. Teil 2: Lexikalische Strukturen. Berlin: de Gruyter.

Haß, Ulrike (ed.) (2005): Grundfragen der elektronischen Lexikographie. elexiko - das OnlineInformationssystem zum deutschen Wortschatz. Berlin: de Gruyter.

Haß, Ulrike / Schmitz, Ulrich (2010): «Lexikographie im Internet 2010 - Einleitung», en Ulrike Haß / Ulrich Schmitz, eds., Thematic Part: Lexikographie im Internet 2010, Lexicographica, 26/2010, Berlin/New York: de Gruyter, 1-18.

Hausmann, Franz J. (1990a): «Das Synonymenwörterbuch. Die kumulative Synonymik?», en Franz J. Hausmann et al., eds., Dictionaries. An International Encyclopedia of Lexicography. HSK 5.2, Berlin: de Gruyter, 1076-1081.

- (1990b): «Das Antonymenwörterbuch», en Franz J. Hausmann et al., eds., Dictionaries. An International Encyclopedia of Lexicography. HSK 5.2, Berlin: de Gruyter, 1081-1083.

- (1991): «Die zweisprachige Lexikographie Spanisch - Deutsch, Deutsch - Spanisch», en Franz J. Hausmann et al., eds., Dictionaries. An International Encyclopedia of Lexicography. HSK 5.3. Berlin: de Gruyter, 2987-2991.

Hausmann, Franz J. et al. (eds.) (1989-1991): Dictionaries. An International Encyclopedia of Lexicography. HSK 5.1-5.3. Berlin: de Gruyter. 
Hausmann, Franz J. / Werner, Reinhold (1991): "Spezifische Bauteile und Strukturen zweisprachiger Wörterbücher: eine Übersicht", en Franz J. Hausmann et al., eds., Dictionaries. An International Encyclopedia of Lexicography. HSK 5.3, 2729-2770.

Honnef-Becker, Irmgard (2002): «Die Benutzung des de Gruyter Wörterbuches Deutsch als Fremdsprache in Situationen der Textproduktion", en Herbert E. Wiegand, ed., Perspektiven der pädagogischen Lexikographie des Deutschen II. Untersuchungen anhand des „de Gruyter Wörterbuchs Deutsch als Fremdsprache“. Tübingen: Niemeyer, 623-646.

Klein, Wolfgang / Geyken, Alexander (2010): «Das Digitale Wörterbuch der Deutschen Sprache (DWDS)", en Ulrike Haß / Ulrich Schmitz, eds., Thematic Part: Lexikographie im Internet 2010. Lexicographica, 26/2010, Berlin/New York: de Gruyter, 79-96.

Klosa, Annette (ed.) (2008): "Lexikographische Portale im Internet», OPAL-Sonderheft 1/2008, IDS-Mannheim [online publizierte Arbeiten zur Linguistik]. Disponible en <http://pub.ids-mannheim.de/laufend/opal/pdf/opal2008-1.pdf> [Consulta: 01/03/ 2014].

- (2009): «Modern German Dictionaries and Their Impact on Linguistic Research», en Silvia Bruti et al., eds., Perspectives on Lexicography in Italy and Europe. Newcastle: Cambridge Scholars Publishing, 175-199.

Konerding, Klaus P. (1998): «Die semantischen Angaben in LANGENSCHEIDTS GRoßörTERBUCH DeUTSCH ALS FREMDSPRACHE», en Herbert E. Wiegand, ed., Perspektiven der pädagogischen Lexikographie des Deutschen. Untersuchungen anhand von "Langenscheidts Großwörterbuch Deutsch als Fremdsprache“. Tübingen: Niemeyer, 107143.

Korhonen, Jarmo (ed.) (2001): Von der mono- zur bilingualen Lexikographie für das Deutsche. Frankfurt: Peter Lang.

Köster, Lutz / Neubauer, Fritz (2002): «Kollokationen und Kompetenzbeispiele im DE GruYter WÖrterbuCh DeUTSCH ALS FremdSPRACHE», en Herbert E. Wiegand, ed., Perspektiven der pädagogischen Lexikographie des Deutschen II. Untersuchungen anhand des „de Gruyter Wörterbuchs Deutsch als Fremdsprache“. Tübingen: Niemeyer, 283-310.

Lehr, Andrea (1998): «Kollokationen in LANGENSCHEIDTS GROßWÖRTERBUCH DEUTSCH ALS FREMDSPRACHE", en Herbert E. Wiegand, ed., Perspektiven der pädagogischen Lexikographie des Deutschen. Untersuchungen anhand von "Langenscheidts Großwörterbuch Deutsch als Fremdsprache". Tübingen: Niemeyer, 256-281.

Mann, Manfred (2010): «Internet-Wörterbücher am Ende der „Nulljahre“», en Ulrike Haß / Ulrich Schmitz, eds., Thematic Part: Lexikographie im Internet 2010, Lexicographica, 26/2010. Berlin/New York: de Gruyter, 19-45.

Meliss, Meike (2005): Recursos lingüísticos alemanes relaticos a 'GERÄUSCH' y sus posibles correspondencias en español. Un estudio lexicológico modular-integrativo. Frankfurt: Peter Lang

- (2011): «Wörterbücher von heute und morgen. Überlegungen zu lexikographischen Benutzersituationen im zweisprachigen Kontext: Spanisch-Deutsch", en $M^{a}$ José Domínguez Vázquez / Emilio González Miranda / Meike Meliss / Víctor Millet, eds., La palabra en el texto. Santiago de Compostela: Universidade de Santiago de Compostela, 267300.

- (2013a): «Das zweisprachige Wörterbuch im bilateralen deutsch-spanischen Kontext. Alte und neue Wegem, en $\mathrm{M}^{\mathrm{a}}$ José Domínguez Vázquez, ed., Trends in der Lexikographie. Frankfurt: Peter Lang, 61-87.

- (2013b): «Online-Lexikographie im DaF-Bereich: Eine erste kritische Annäherung: Bestandsaufnahme - Nutzen - Perspektiven", Real Revista de Estudos Alemães, 4, 176-199. Disponible en <http://real.fl.ul.pt/textos.page/ pag/2> [Consulta: 11/04/2014].

- (2014a): «(Vor)überlegungen zu einem zweisprachigen Produktionslernerwörterbuch für das Sprachenpaar DaF und ELEm, en Daniel Reimann, ed., Kontrastive Linguistik und 
Fremdsprachendidaktik Iberoromanisch-Deutsch. Studien $z u$ Morphosyntax, Mediensprache, lexikographie und mehrsprachigkeitsdidaktik. Tübingen: Narr, 113-137.

- (2014b): «Das verbale Kombinationspotenzial in einsprachigen DaF-Lernerwörterbüchern: Kritische Bestandsaufnahme - Neue Anforderungen", ZDaF 4/2014 (en prensa).

Meliss, Meike / Sánchez Hernández, Paloma (2014): "Theoretical and methodological foundations of the DICONALE project: a conceptual dictionary of German and Spanish", en João Paulo Silvestre / Alina Villalva, eds., Planning non existent dictionaries. Dicionarística Portuguesa. IV. Lisboa: CLUL (en prensa).

Meyer, Meike / Wiegand, Herbert E. (2000): «Gemischt-semintegrierte Mikrostrukturen für deutsch-spanische Printwörterbücher", en Herbert E. Wiegand, ed., Studien zur zweisprachigen Lexikographie mit Deutsch V. Hildesheim: Olms, 87-171.

Model, Benedikt (2010): Syntagmatik im zweisprachigen Wörterbuch. Berlin: de Gruyter.

Müller-Spitzer, Caroline / Engelberg, Stefan (2011): "Elektronische Lexikographie zwischen Grammatik und Lexikon", en Stefan Engelberg et al., eds., Sprachliches Wissen zwischen Lexikon und Grammatik. Berlin: de Gruyter, 559-572.

- (2013): «Dictionary Portals», HSK, 4, Lexikographie, 1023-1035.

Porto Dapena, José-Alvaro / Conde Noguerol, Eugenia / Córdoba Rodríguez, Félix / Muriano Rodríguez, Ma Montserrat (2007): «El diccionario "Coruña" de la lengua española actual: planta y muestra", Anexos de Revista de lexicografia, 9. A Coruña: Servicio de Publicaciones.

- (2008): «Presentación del diccionario "Coruña" de la lengua española actual», en Elisende Bernal / Janet A. de Cesaris, eds., Proceedings of the Xiii Euralex International Congress. Barcelona: Documenta Universitaria, Série Activitats, 20, 753-762.

Pöll, Bernhard (2010): «Internetlexikographie der iberomanischen Sprachen», en Ulrike Haß / Ulrich Schmitz, eds., Thematic Part: Lexikographie im Internet 2010, Lexicographica, 26/2010, Berlin/New York: de Gruyter, 169-173.

Proost, Kristel (2007): Conceptual structure in lexical items: The lexicalisation of communication concepts in English, German and Dutch. Amsterdam/Philadelphia: Benjamins.

Roelcke, Thorsten (2002): «Das Verhältnis der semasiologischen und onomasiologischen Angaben im de Gruyter Wörterbuch Deutsch als Fremdsprachem, en Herbert E. Wiegand, ed., Perspektiven der pädagogischen Lexikographie des Deutschen II. Untersuchungen anhand des "de Gruyter Wörterbuchs Deutsch als Fremdsprache“. Tübingen: Niemeyer, 201-244.

Ruhstaller, Stefan / Gordón, Ma Dolores (eds.) (2010): Diccionario y aprendizaje del español. Frankfurt: Peter Lang.

Sanmarco Bande, $M^{a}$ Teresa (2008): «La lexicografía del siglo XXI: hacia el diccionario ilimitado", en Mar Campos Souto et al., eds., La lexicografía bilingüe y didáctica: ayer y hoy. Anexos Revista de Lexicografía, 8. La Coruña: Universidade da Coruña, 133-140.

Schafroth, Elmar (2002): «Die Grammatik der Verben im DE GRUYTER WÖRTERBUCH DEUTSCH ALS FREMDSPRACHE", en Herbert E. Wiegand, ed., Perspektiven der pädagogischen Lexikographie des Deutschen II. Untersuchungen anhand des "de Gruyter Wörterbuchs Deutsch als Fremdsprache“. Tübingen: Niemeyer, 57-74.

Storrer, Angelika (2010): «Deutsche Internet-Wörterbücher: Ein Überblick», en Ulrike Haß / Ulrich Schmitz, eds., Thematic Part: Lexikographie im Internet 2010, Lexicographica, 26/2010, Berlin/New York: de Gruyter, 154-164.

Tarp, Sven (2012): «Online dictionaries: today and tomorrow», en Ulrich Heid, ed., Thematic Part: Corpora and Lexicography, Lexicographica, 28/2012. Berlin/New York: de Gruyter, 253-267. 
Torres del Rey, Jesús (2009): «Diccionarios electrónicos bilingües: nuevas posibilidades de futuro", en Ma Teresa Fuentes Morán / Benedikt Model, eds., Investigaciones sobre lexicografía bilingüe. Granada: Tragacanto, 29-79.

Werner, Reinhold (1998): "La selección de lemas en los diccionarios español-alemán y alemánespañol o ¿un diccionario de qué lengua es un diccionario de las lenguas española y alemana?n, en $\mathrm{M}^{\mathrm{a}}$ Teresa Fuentes Morán / Reinhold Werner, eds., Lexicografias iberrománicas: problemas, propuestas y proyectos. Frankfurt: Vervuert, 139-156.

Wiegand, Herbert E. (ed.) (1998): Perspektiven der pädagogischen Lexikographie des Deutschen. Untersuchungen anhand von "Langenscheidts Großwörterbuch Deutsch als Fremdsprache“. Tübingen. Niemeyer.

- (ed.) (2002): Perspektiven der pädagogischen Lexikographie des Deutschen II. Untersuchungen anhand des "de Gruyter Wörterbuchs Deutsch als Fremdsprache“. Tübingen. Niemeyer.

- (2005): «Äquivalentpräsentation und Wörterbuchfunktion in zweisprachigen Printwörterbüchern. Mit einem Seitenblick auf die so genannte 'moderne lexikographische Funktionslehre'", en Birgit Igla / Pavel Petkov / Herbert E. Wiegand, eds., Kontrastive Lexikologie und zweisprachige Lexikographie. Hildesheim: Olms, 1-38.

Wolf, Norbert R. (2001): «Kollokationen und semantische Valenz im einsprachigen Wörterbuch", en Jarmo Korhonen, ed., Von der mono- zur bilingualen Lexikographie für das Deutsche. Frankfurt: Peter Lang, 153-162.

Wolski, Werner (2002): «Das de Gruyter Wörterbuch Deutsch als Fremdsprache und Langenscheidts Großwörterbuch Deutsch als Fremdsprache. Ein Vergleich im Hinblick auf die Semantik», en Herbert E. Wiegand, ed., Perspektiven der pädagogischen Lexikographie des Deutschen II. Untersuchungen anhand des "de Gruyter Wörterbuchs Deutsch als Fremdsprache“. Tübingen: Niemeyer, 3-34. 